\title{
Use of molecular genetic markers for the prediction of clinical course of ovarian hyperstimulation syndrome
} \author{
Abubakirov Aydar ${ }^{1}$ \\ Research centre of obstetrics, gynaecology and perinatology \\ named after academician V.I. Kulakov, Moscow, Russia \\ ${ }^{1}$ Department of preservation and restoration \\ of reproductive function \\ ${ }^{2}$ Laboratory of molecular genetic methods
}

Strelchenko Daria ${ }^{1}$, Perminova Svetlana1, Donnikov Andrey ${ }^{2}$, Korneeva Irina ${ }^{1}$,

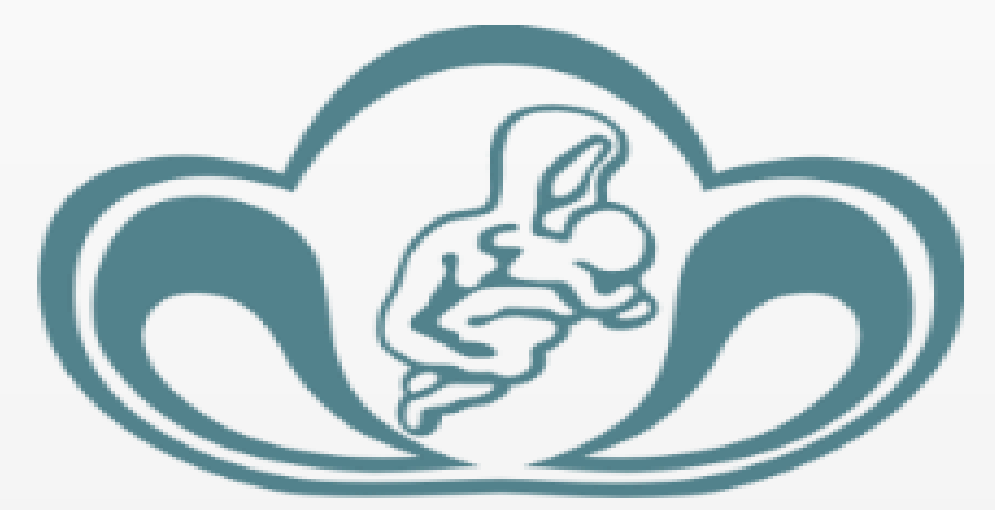

The need for OHSS prevention in individual patients is usually assessed based on clinical risk factors, for example the levels of $\mathrm{AMH}$, FSH, antral follicle count (AFC), etc. However, preventative strategies for OHSS fail in some patients who still develop the complication. This has led to a hypothesis that finer molecular factors such as genetic polymorphisms may predispose patients to OHSS, and the risks of this complication may not be predicted fully until molecular genetics tools are routinely applied in the clinical practice.

A case-control study including 287 women was carried out from November 2013 till May 2016. Fifty-nine patients from both groups developed clinically relevant OHSS (Group 1 - early OHSS, $n=41$; Group 2 - late OHSS; $\mathrm{n}=18$ ).

Inclusion criteria for Group 1 included presence of clinical symptoms of OHSS 3 to 9 days after ovulation trigger injection. For inclusion into Group 2, clinical symptoms of OHSS had to manifest 9 to 12 days after ovulation trigger injection. Gene polymorphisms were identified using qPCR with the following gene loci: IL18, ICAM1, VEGFA, BSG, AMH, IL2, LHCGR, EDN1, IL6, AMHR2, EDNRA, FSHR, IL1R1, INHA, ESR1, TNF, IL1B, ESR2, TSHR, IL8, ACE. To predict the onset of early and late OHSS, we performed a ROC analysis of the association between the early and late OHSS and general clinical and laboratory parameters. Early OHSS was significantly associated with AMH (AUC - 0,677 (95\% 0,602-0,751) and AFC (AUC - 0,664 (95\% 0,585-0,742)). We established that the AMH threshold of 3.7 $\mathrm{ng} / \mathrm{ml}$ and AFC threshold of 14 follicles had an $82 \%$ and $84 \%$ sensitivity and $54 \%$ and $47 \%$ specificity in predicting early OHSS, respectively. ROC analysis of association between late OHSS and clinical and laboratory parameters has not revealed any significant predictors.

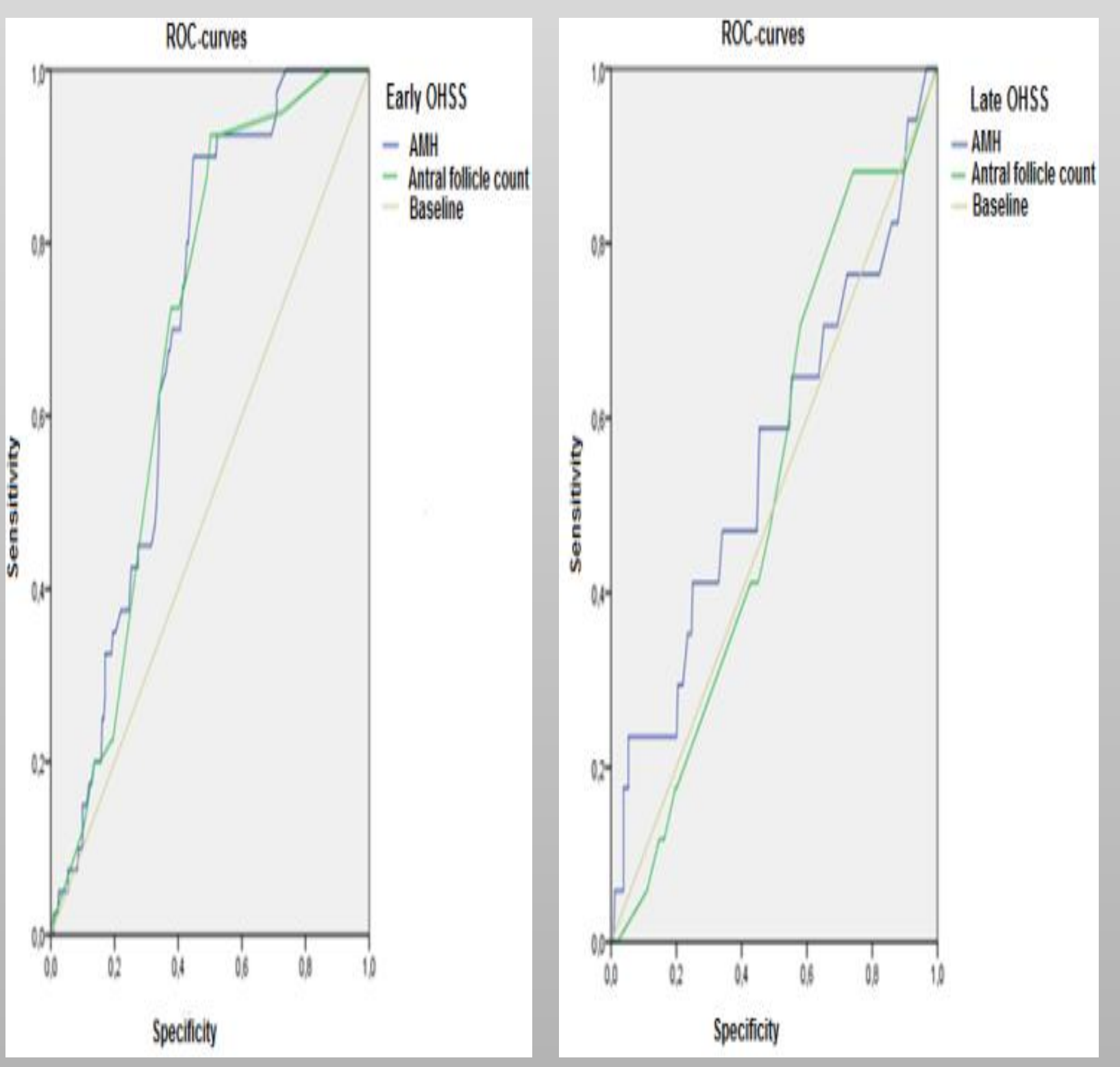

Figure 1
To predict the development of early and late OHSS, a search for genetic markers associated with OHSS onset was conducted. According to the binary logistic regression modeling, only four genotype variables could be used to predict early OHSS. These included the VEGFA -2578(-2595) A $>$ C [rs699947], VEGFA $936 \mathrm{C}>\mathrm{T}$ [rs3025039], LHCGR 935 A>G (Asn312Ser) [rs 2293275], and ESR1 $351 \mathrm{~A}>\mathrm{G}$ [Xbal] [rs9340799] genotypes.

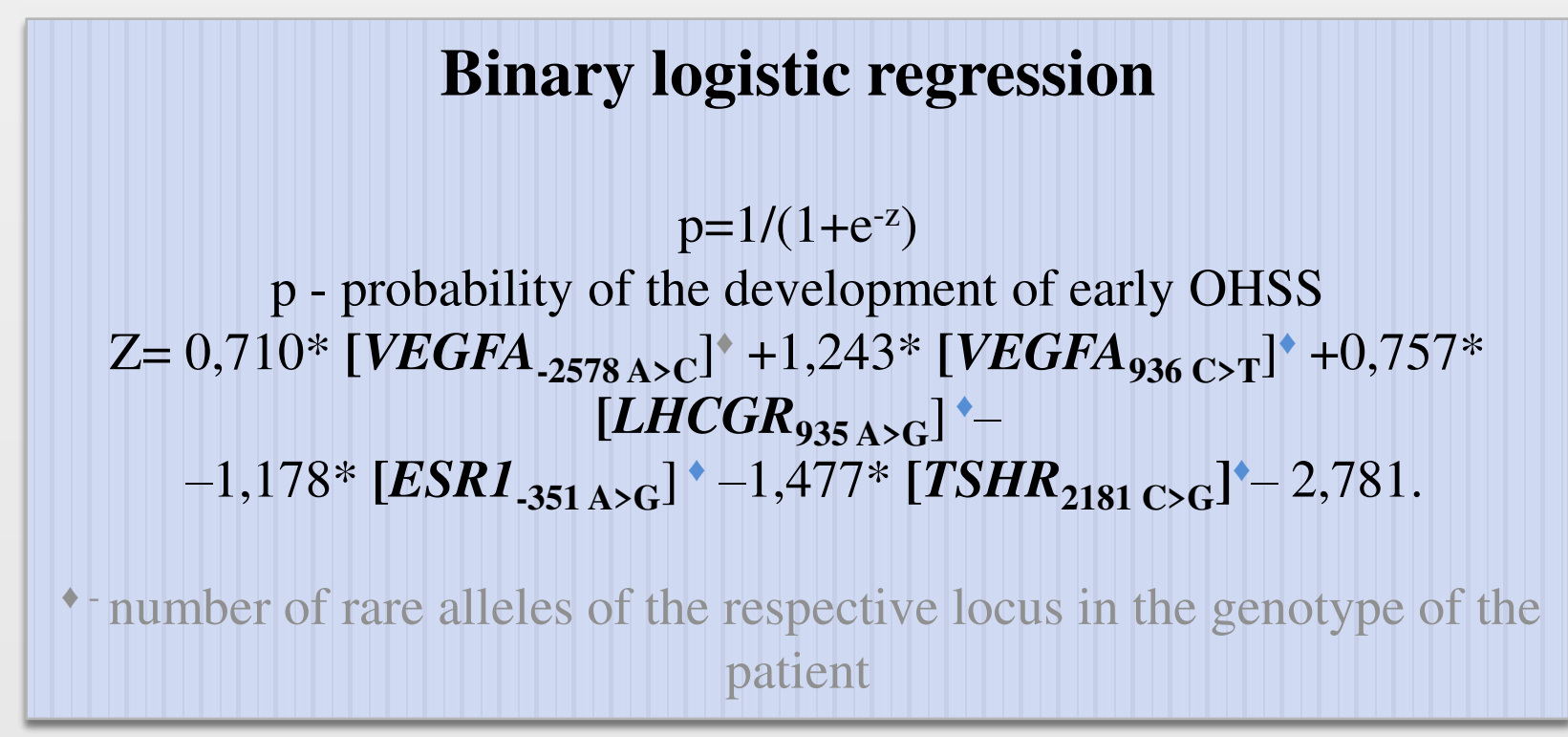

Another two genotype variables allowed to predict late OHSS (ESR1 -351 A>G [Xbal] [rs9340799] and ACE 287bp Ins>Del $((\mathrm{ALU}) /-)[\mathrm{rs} 4340])$.

\section{Binary logistic regression}

$\mathrm{p}=1 /\left(1+\mathrm{e}^{\mathrm{z}}\right), \mathrm{p}-$ probability of the development of late OHSS $\mathrm{Z}=1,811^{*}\left[\boldsymbol{E S R I}_{-351 \mathrm{~A}>\mathrm{G}}\right]^{\star}-1,973 *\left[\boldsymbol{T S H R}_{2181 \mathrm{C}>\mathrm{G}}\right]^{\dagger}+1,351 *[\boldsymbol{A C E}$ 287bp Ins>Del $]^{\star}-6,641$.

number of rare alleles of the respective locus in the genotype of the patient

The C/C genotype of TSHR:2181C $>$ G (Asp727Glu) [rs1991517] was significantly associated with OHSS onset $(\mathrm{OR}=1.96(95 \% \mathrm{CI}$ (0.94-4.11); $\mathrm{p}=0.042)$. Genetic factors contributed approximately $25 \%$ to the development of early and late forms of OHSS (calculated by Nagelkerke method).

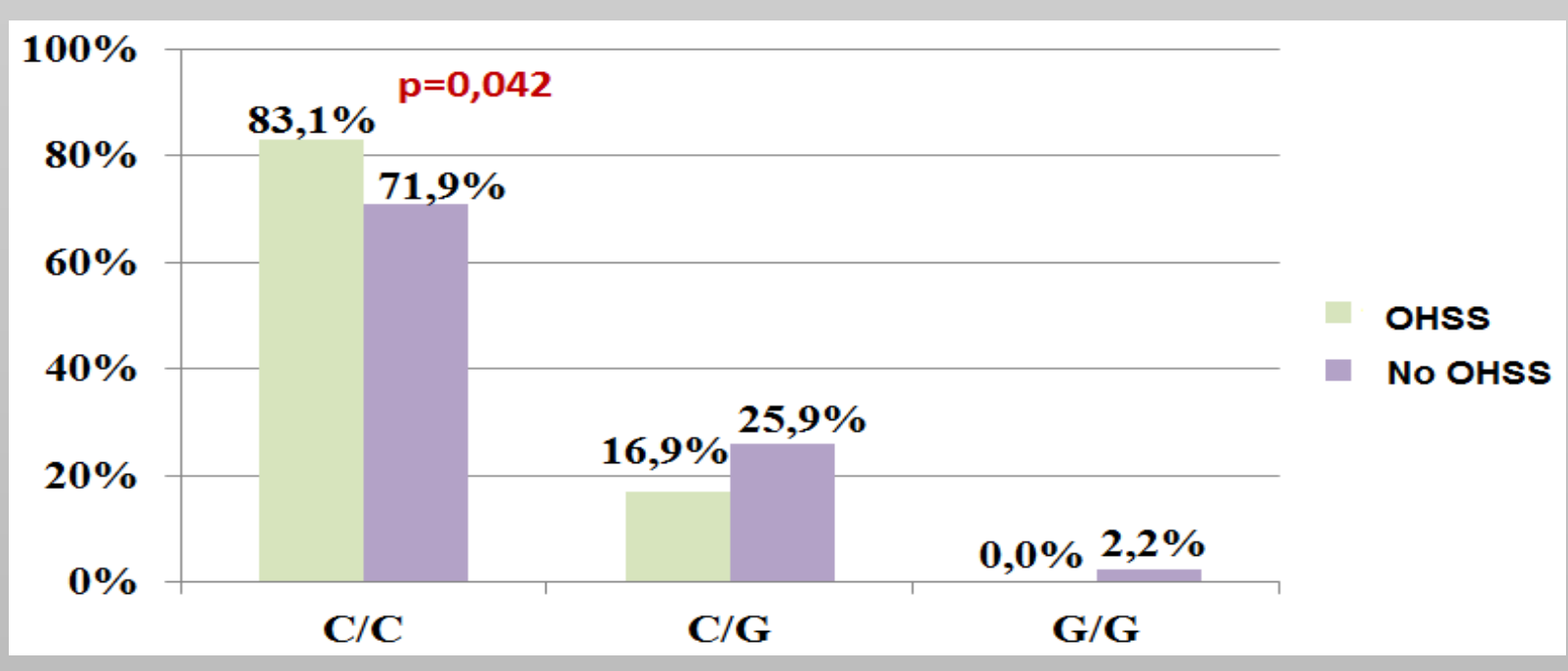

Figure 2

This study opens new possibilities for OHSS prediction as it demonstrated the association between the clinical course of OHSS and the patient's genotype. Combined evaluation of clinical and genetic predictors will allow us to tailor ovarian stimulation and, consequently reduce the OHSS risks. 